\title{
Extent of Cerebrovascular Disruption Following Blast Exposure is Influenced by the Duration of the Positive Phase in Addition to Peak Overpressure
}

Dexter V Reneer ${ }^{1,2}$, Carolyn A Crowdus ${ }^{1,2}$, Sarbani Ghoshal ${ }^{1}$, Julie Corkins ${ }^{1}$, Richard D Hisel ${ }^{3}$, Braden T Lusk ${ }^{4}$ and James W Geddes ${ }^{1,2 *}$

${ }^{1}$ Spinal Cord and Brain Injury Research Center, College of Medicine, University of Kentucky, Lexington, KY, USA

${ }^{2}$ Department of Anatomy and Neurobiology, College of Medicine, University of Kentucky, Lexington, KY, USA

${ }^{3}$ Department of Biostatistics, College of Public Health, University of Kentucky, Lexington, KY, USA

${ }^{4}$ Department of Mining Engineering, College of Engineering, University of Kentucky, Lexington, KY, USA

\begin{abstract}
Blast-induced traumatic brain injury (bTBI) has been described as the defining injury of Operations Enduring Freedom and Iraqi Freedom (OEF/OIF). Although there has been a significant amount of research characterizing the brain injury produced by blast, greater understanding of the contribution of each component of the shockwave to the injury is needed. Large animal models of bTBI utilize chemical explosives as their shockwave source while small animal models predominantly utilize compressed air-driven membrane rupture as their shockwave source. We previously designed and built a multi-mode shock tube capable of utilizing air-driven membrane rupture or chemical explosives (oxyhydrogen: A 2:1 mixture of hydrogen and oxygen gasses to produce a shockwave. Compressed airdriven shockwaves exhibited longer duration positive phases than compressed oxyhydrogen-driven shockwaves of similar peak overpressure. The longer duration of compressed air-driven shockwaves results in greater energy being imparted on a test subject than would be impacted by shockwaves of identical peak overpressures from the other sources. Animals exposed to compressed air-driven shockwaves exhibited more extensive brain surface hematoma and more blood-brain barrier compromise than did animals exposed to oxyhydrogen-driven shockwaves of even greater peak overpressure. Taken together, these data suggest that compressed air-driven shockwaves contain more energy than their chemical explosive-derived counterparts of equal peak overpressure and can result in greater injury in an experimental animal model. Additionally, these data suggest that exposure to longer duration shockwaves can result in more severe bTBI. The results of this study are relevant to the design of blast wave mitigation technology and future clinical intervention.
\end{abstract}

Keywords: Traumatic brain injury; Blood-brain barrier; Immunoglobulin; Shock tube; Rats3

\section{Introduction}

Secondary injury mechanisms associated with TBI pathology are often a significant source of damage and dysfunction in addition to the primary mechanical trauma. One significant consequence of sustaining a TBI is vascular damage. Physical disruption of brain vasculature can lead to hematoma and hemorrhage within the cranial vault.

Vasogenic edema caused by compromise to the blood-brain barrier (BBB) giving rise to leaky blood vessels following TBI often leads to significant accumulation of extravasated fluid within the brain parenchyma [1]. Further edema through a vasogenic response to cytotoxic mechanisms leads to a cyclical downward progression of the injured brain's ability to recover [2]. Regional changes in blood flow and changes in blood-brain barrier permeability have been reported in rodent models of diffuse [3-5] and focal [6] models of traumatic brain injury. Current treatments are aimed at reducing intracranial pressure and, thus, increasing cerebral perfusion pressure and breaking the cycle of edema, however these treatments work through reducing osmotic pressure and do not treat the source of the BBB problem. Nevertheless, it is important to determine the contribution of $\mathrm{BBB}$ compromise in models of traumatic brain injury.

Reported peak pressures necessary to produce blast injury have varied greatly from $20 \mathrm{kPa}$ [7] for whole-body exposure to 20,000 kPa for direct brain exposure to shockwaves [8]. These reports suggest that it is not simply the magnitude of blast overpressure, but also additional components of the blast shockwave that contribute to the resultant brain injury.
We developed and characterized a rat model of blast injury that utilizes a multi-mode shock tube (McMillan Blast Device; MBD) to produce shockwaves with different unique pressure-time signatures [9]. This device can use compressed-air or other driving sources including oxyhydrogen to produce a blast wave. The relatively long overpressure durations produced by 4 ] are similar to those resulting from exposure at a distance from a huge chemical explosion $(>20,000$ $\mathrm{kg}$ TNT) or nuclear blast $[10,11]$. In contrast, the shorter overpressure duration produced using oxyhydrogen as the driving source closely resembles the blast wave produced by produced by plastic explosives and stacked artillery shells typical of IEDs [9].

Using the MBD, we are able to compare results obtained using compressed-air driven and oxyhydrogen driven blast shock waves. Moreover, the design of our shock tube satisfies criteria outlined by Bell [12] for modeling bTBI in animals. Placing the animal within the shock tube is optimal for simulating primary blast injury $[13,14]$.

*Corresponding author: James W Geddes, Spinal Cord and Brain Injury Research Center, B477 Biomedical \& Biological Sciences Research Building (BBSRB) 741. S. Limestone Street, University of Kentucky Lexington, KY 40536-0509; Tel: 859323-5135; Fax: 859-257-5737; E-mail: jgeddes@uky.edu2

Received November 01, 2013; Accepted January 24, 2014; Published February 05, 2014

Citation: Reneer DV, Crowdus CA, Ghoshal S, Corkins J, Hisel RD, et al. (2014) Extent of Cerebrovascular Disruption Following Blast Exposure is Influenced by the Duration of the Positive Phase in Addition to Peak Overpressure. J Neurol Neurophysiol 5: 188. doi:10.4172/2155-9562.1000188

Copyright: (c) 2014 Reneer DV, et al. This is an open-access article distributed under the terms of the Creative Commons Attribution License, which permits unrestricted use, distribution, and reproduction in any medium, provided the original author and source are credited. 
Citation: Reneer DV, Crowdus CA, Ghoshal S, Corkins J, Hisel RD, et al. (2014) Extent of Cerebrovascular Disruption Following Blast Exposure is Influenced by the Duration of the Positive Phase in Addition to Peak Overpressure. J Neurol Neurophysiol 5: 188. doi:10.4172/21559562.1000188

Placing the rat within a Kevlar vest and then within a smaller steel tube with the head exposed also protects internal organs, as determined by necropsy following blast exposure [9]. Following the observation by Goldstein and colleagues that blast waves can cause rotational head oscillations which may contribute to the brain injury [15], we used high speed video to record head movement during blast exposure. Although the angle and velocity of head rotation did not differ between the two blast sources, the results support the possible contribution of a tertiary (hyperacceleration and head rotation) mechanism to the brain injury in addition to the primary blast injury resulting directly from the blast shock wave.

In the initial characterization of this device, we reported that rats exposed to compressed-air driven blasts appeared to exhibit more prominent vascular damage than was observed following oxyhydrogen-driven blasts of similar peak overpressures, based on gross pathology [9]. In the present study, we examined the brains of rats exposed to compressed air-driven shockwaves of $175 \mathrm{kPa}$ peak target overpressure and oxyhydrogen-driven shockwaves of $250 \mathrm{kPa}$ peak target overpressure for evidence of blood brain-barrier compromise at 3 hours post blast exposure. This 5 time point was chosen to coincide with the time at which maximal IgG extravasation has been reported to occur in other models of blast-induced traumatic brain injury [16,17].

We also examined extravasated IgG levels following air-driven blast shockwaves of peak overpressures ranging from 125 to $175 \mathrm{kPa}$. The results demonstrate that the duration of the positive phase of the blast shockwave, in addition to peak overpressure, is an important determinant of blast-induced brain injury.

\section{Materials and Methods}

\section{Animals and blast shockwave exposure}

All animal use procedures utilized in this study were performed in accordance with a protocol approved by the University of Kentucky Institutional Animal Care and Use Committee. Animals had access to food and water ad libitum throughout the course of this study. Adult (8week), male Sprague-Dawley Rats (Harlan Laboratories, Indianapolis, IN, USA) were separated into individual animal cages, sedated (diazepam, $4 \mathrm{mg} / \mathrm{kg}$, i.p.), and transported via climate controlled passenger van from the animal housing facility to the blast site. At the blast site, the animals were maintained in a climate-controlled room with adequate lighting for the duration of their time at the blast site. Immediately prior to injury, animals were deeply anesthetized (ketamine $60 \mathrm{mg} / \mathrm{kg}$, xylazine $7.5 \mathrm{mg} / \mathrm{kg}$, i.p.), fitted with a Kevlar vest, placed within polyethylene netting (Industrial Netting, Minneapolis, MN, USA), and loaded into the McMillan Blast Device (MBD) [9] in the prone position with their left side facing the shockwave source. Animals were subjected to compressed air- or oxyhydrogen-driven blasts of $175 \mathrm{kPa}$ or $250 \mathrm{kPa}$ peak overpressure, respectively. Animals were removed immediately following blast [9]. Following blast injury, animals were returned to their individual cages with heat support (Deltaphase Isothermal Pads, Braintree Scientific, Braintree, MA, USA) to prevent anesthesia-induced hypothermia. Animals were maintained in dorsal recumbency for the post-blast anesthesia period and were periodically monitored for respiration. Cardiac activity was monitored via thoracic palpation. Due to the fact that headache is known to occur in humans exposed to blast, rats were weighed and given carprofen (7 $\mathrm{mg} / \mathrm{kg}$, i.p.) after blast exposure. Once recovered from anesthesia, all animals were placed in clean individual cages and transported back to the University of Kentucky animal facility where they remained in their individual cages until euthanasia.

\section{Euthanasia and tissue collection}

At $3 \mathrm{~h}$ post injury animals were administered an overdose of sodium pentobarbital $(150 \mathrm{mg} / \mathrm{kg}$, i.p.). This time point was chosen based on known peak BBB opening time following other modes of traumatic brain injury [16-18]. Once animals were deeply anesthetized, thoracotomy was performed and animals were transcardially perfused with $200 \mathrm{~mL}$ of phosphate-buffered saline (PBS) to remove blood from the vasculature, followed by $200 \mathrm{~mL}$ of $4 \%$ paraformaldehyde in PBS for initial in situ tissue fixation. Animals used for Western blot analysis were perfused only with PBS. After perfusion, animal brains were removed and photographed for evidence of hematoma and petechial hemorrhage. Brains used for immunohistochemistry were placed in $4 \%$ paraformaldehyde in PBS overnight at $4^{\circ} \mathrm{C}$, followed by three days in $30 \%$ sucrose in $\mathrm{PBS}$ at $4^{\circ} \mathrm{C}$ for cryoprotection, after which they were embedded in OCT compound and stored at $-80^{\circ} \mathrm{C}$ until sectioning. Brains used for Western blot analysis were separated into cerebrum and brainstem/cerebellum sections by severing the cerebral peduncles and brainstem-diencephalon connections. The cerebrum was further subdivided by a midsaggital bisection creating left and right cerebrum samples. The left and right cerebrum samples were further subdivided into dorsal and ventral sections by horizontal cuts just ventral to each hippocampus. These cuts resulted in four cerebrum samples for each animal: ipsilateral ventral cortex (IVX), ipsilateral dorsal cortex (IDX), contralateral ventral cortex (CVX), and contralateral dorsal cortex (CDX). Brainstem (BS) and cerebellum (CBM) were separated by severing the cerebellar peduncles. Each sample was wrapped in aluminum foil, flash frozen in powdered dry ice, and kept frozen at $-80^{\circ} \mathrm{C}$ until processing for protein.

\section{Immunohistochemistry}

Brains were sectioned at $40 \mu \mathrm{m}$ thickness on a freezing stage sliding microtome (Microm HM 440 E, Microm, Walldorf, Germany). Sections were collected in Tris-buffered Saline (TBS). Sections representative of four different anterior-posterior levels were matched across animals and blocked for 1 hour in $1 \%$ horse serum in TBS and treated in primary antibody in $1 \%$ horse serum in TBS for 1 hour at room temperature. Antibody against immunoglobulin G (IgG, Goat ant-Rat IgG-Biotin, 1:5,000, Jackson Immunoresearch, West Grove, PA, USA) was diluted in TBS with $1 \%$ horse serum and applied to the sections overnight at room temperature. Biotinylated antibody treated slices were treated with streptavidin-conjugated horseradish peroxidase (1:5,000, Jackson Immunoresearch). Presence of antibody reactivity was detected via enzymatic oxidation of 3,3'-diaminobenzidine (DAB, Jackson Immunoresearch). Following staining, slides were mounted on to slides (Fisher SuperFrost Plust, Fisher Scientific, Waltham, MA, USA) and mounted with Permount (Fisher Scientific) and coverslips. Slides were imaged on an Olympus AX 80 microscope. IgG images were analyzed using Image J software for the percentage area reactive to IgG. Of the four anterior-posterior levels matched between all animals, the level with the most consistent IgG staining between all animals in a given treatment group was chosen for the Image $J$ analysis. Captured images were subjected to binary isodataalgorithm threshold analysis to quantify percent area covered by IgG immunoreactivity. Any pixel that crossed threshold was assigned the color black and any pixel that failed to cross threshold was assigned the color white. Percentage brain section area positive for black pixels was recorded and averaged. Areas counted as IgG positive and/or part of the section area but that were part of the ventricles were subtracted from the total.

\section{Western blotting}

Frozen brain tissue was weighed, covered with $150 \mu \mathrm{l}$ protein and 
Citation: Reneer DV, Crowdus CA, Ghoshal S, Corkins J, Hisel RD, et al. (2014) Extent of Cerebrovascular Disruption Following Blast Exposure is Influenced by the Duration of the Positive Phase in Addition to Peak Overpressure. J Neurol Neurophysiol 5: 188. doi:10.4172/21559562.1000188

RNA isolation buffer; $(50 \mathrm{mM}$ Tris, $150 \mathrm{mM} \mathrm{NaCL}, 1 \mathrm{mM}$ EDTA, 0.5\% NP-40, 0.5\% Triton-X-100, 5 mM DTT, 1 table Roche complete protease inhibitor tablet $/ 50 \mathrm{ml}$ ) and allowed to thaw on ice. Thawed tissue was chopped, placed in a dounce homogenizer with the above buffer ( $2 \mathrm{ml}$ per $\mathrm{g}$ of tissue), and homogenized by 20 passes in the homogenizer. Resulting lysates were centrifuged at $13,200 \mathrm{rpm}$ for 2 min, the supernatant collected, centrifuged again and the supernatant collected. Protein concentrations were quantified by bicinchoninic acid (BCA) Protein Assay (Thermo Scientific). Lysates were separated by SDS-PAGE (10\% Bis-Tris, Invitrogen) using MOPS Running Buffer (Invitrogen), transferred to nitrocellulose membranes via semidry protein transfer (Trans-Blot Turbo Transfer System, Bio-Rad, Carlsbad, CA, USA). Membranes were blocked in Trisbuffered Saline (TBS) containing $5 \%(\mathrm{w} / \mathrm{v})$ non-fat powdered milk for one hour at room temperature to prevent non-specific antibody-protein interaction. Once blocked, membranes were treated with TBS containing $0.05 \%$ Tween-20 (TTBS) and primary antibody (1:1,000, Sigma) overnight at $4^{\circ} \mathrm{C}$. Membranes were washed 3 times for 5 minutes with TTBS, and then treated with TTBS containing the appropriate secondary antibody conjugated to an infrared fluorphore (1:5,000, Rockland). Following secondary antibody treatments, membranes were washed 3 times for 5 minutes with TTBS, then imaged on an Odyssey Scanner (Li-Cor Biosciences, Lincoln, NE, USA) and analyzed for relative band intensity on the Image Studio software (Li-Cor).

\section{Head rotation analysis}

A three-inch porthole in the blast shocktube was fitted with a Plexiglas window. Rat head movement during the blast exposure was recorded via high-speed (300 frames per second) video camera (Sanyo Xacti HD 1010). Videos were analyzed by an observer who was blind to the injury parameters. The extent of head rotation was estimated from images based on the position of the eyes and the nose is each frame. The velocity of head rotation was determined based on the number of frames from the initial head movement to maximum deflection and the frame rate of the video camera.

\section{Statistical analysis}

Data presented in Figures 3,4 was analyzed by One-Way ANOVA. The Student Newman-Keuls post hoc analysis was selected for comparisons between treatment groups due to its ability to protect against both Type I and Type II statistical error. A Two-Tailed Pearson correlation was used in combination with linear regression analysis to examine the extent of IgG extravasation due to pressure or impulse presented in Figure 5.

\section{Results}

\section{Surface vessel damage and hematoma following blast exposure}

Gross examination of the brains of rats exposed to both oxyhydrogen- and compressed air-driven shockwaves revealed surface vessel damage (Figure 1). The brains of rats exposed to compressed air- and oxyhydrogen-driven shockwaves exhibited large scale hematoma and petechial hemorrhage compared to those from sham-exposed animals. The hematomas were centered on the ventral surface of the brain surrounding the median eminence. Hematoma and petechial hemorrhage were evident on the ventral surface of the brains of 5 of 6 rats exposed to compressed air-driven shockwaves, and 3 of 6 rats exposed to oxyhydrogen-driven shockwaves (Figure 1). While hematoma were evident on the ventral surface of the brains of rats exposed to both modes of the MBD, these hematoma were less extensive and the petechial hemorrhage was less evident in the brains of oxyhydrogen-injured rats.

\section{Histological evidence of blood-brain barrier damage following blast exposure}

In addition to gross pathological evidence of vascular compromise due to blast, brains of rats subjected to oxyhydrogen- and compressed air-driven shockwaves were examined for histological evidence of blood-brain barrier compromise. Brains of rats subjected to compressed air-driven shockwaves showed IgG staining that was specific to the inferior cerebrum on the left side; the side first impacted by the shockwave (Figure 2). Representative slices taken from rat brains subjected to sham injury or to oxyhydrogen-driven shockwaves exhibited no bilaterally asymmetric IgG staining. Periventricular and median eminence IgG staining is normal in uninjured animals as the blood-brain barrier is incomplete in these areas. Images presented in Figure 2 were subjected to binary isodata-algorithm threshold analysis (Figure 3A) to determine which areas of the images crossed threshold. Areas determined to have crossed threshold were considered IgG positive. Quantification of the percentage area staining positive for IgG extravasation revealed a significant increase in IgG staining in brain sections from animals subjected to compressed air-driven shockwaves, but not sham- or oxyhydrogen-exposed animals (Figure 3B). Combined with hematoma and petechial hemorrhage data presented in Figures 1 and 2, the data demonstrate that there is significant blood-brain barrier compromise produced by compressed air-driven shockwaves that is less extensive in animals exposed to oxyhydrogen driven shockwaves.

\section{Shockwave energy dose response}

Rats were exposed to compressed air-driven shockwaves of 125 $\mathrm{kPa}, 150 \mathrm{kPa}$, or $175 \mathrm{kPa}$ peak target pressure. Western blot analysis showed a positive trend of increasing IgG levels present in the IVX region with increasing peak pressure (Figure 4). There was no positive trend IgG levels with increasing pressure in either the CBM or BS regions (Figures $4 \mathrm{D}$ and $4 \mathrm{E}$ ). While sham IgG levels were relatively constant, there was substantial variability in the IgG levels throughout all regions sampled at all pressures (Figures $4 \mathrm{C}$ and $4 \mathrm{E}$ ). The reasons for this variability are unclear.

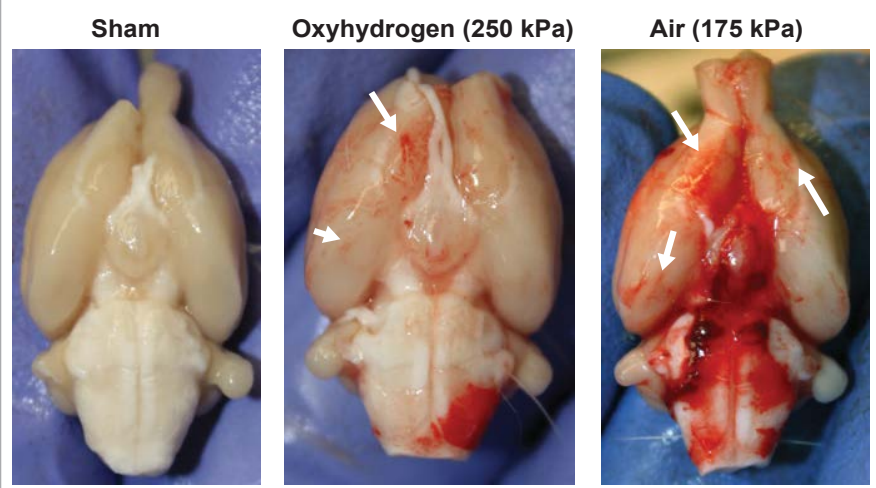

Figure 1: Qualitative examination of vascular damage can be observed in the images above depicting hematoma and petechial hemorrhage on the ventral surface of brains taken at 3 hours post-injury from rats exposed to oxyhydrogen- or compressed air-driven shockwaves. Note larger hematoma and more extensive petechial hemorrhage (white arrows) in the brain from a compressed air-driven shockwave-exposed rat. Images are representative of six rats per treatment group. 
Citation: Reneer DV, Crowdus CA, Ghoshal S, Corkins J, Hisel RD, et al. (2014) Extent of Cerebrovascular Disruption Following Blast Exposure is Influenced by the Duration of the Positive Phase in Addition to Peak Overpressure. J Neurol Neurophysiol 5: 188. doi:10.4172/21559562.1000188
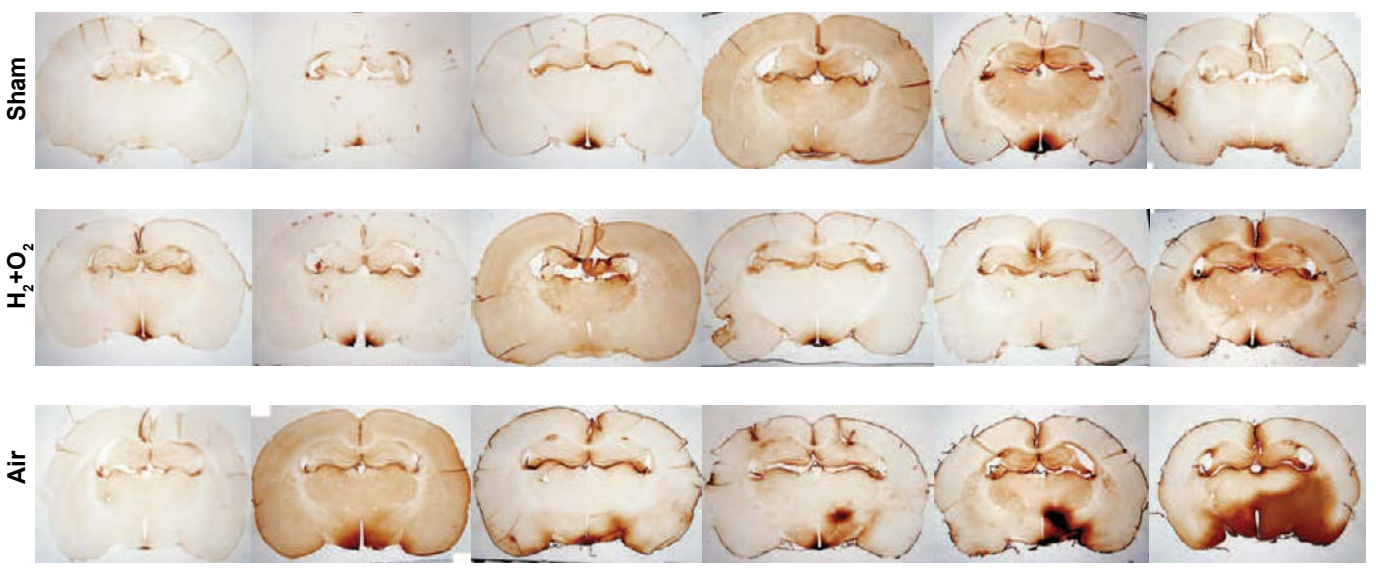

Figure 2: Histological evidence of blood-brain barrier compromise following compressed airdriven shockwave exposure. IgG extravasation can be visualized in the inferior left (side of the shockwave source) side of the brains in 5 of 6 rats exposed to compressed air-driven shockwaves. This region specific staining is not evident in the brains of rats exposed to sham injury or oxyhydrogen-driven shockwaves. Brain sections are each from a different animal euthanized at 3 hours post-injury and are presented in order from least severe to most severe IgG staining. $n=6$ per treatment.



$\mathbf{B}$

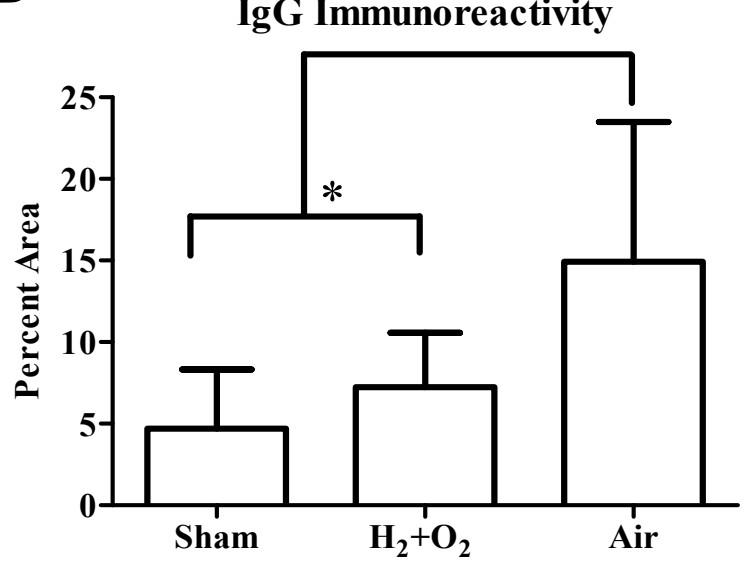

Figure 3: Quantification of IgG Immunoreactivity. (A) A section stained with anti-IgG antibody and subjected to thresholding as described in Methods. This section is from an animal exposed to a compressed-air driven blast, and corresponds to the third section from the left in the bottom row of Figure 2. (A). The percentage area of the slice that was positive for IgG immunoreactivity was averaged. Brains from rats exposed to compressed air-driven shockwaves exhibited more area immunoreactive to IgG than sham- or oxyhydrogen-driven blast-exposed rats at $3 \mathrm{~h}$ post-injury. (B). One-way 21 ANOVA with Student Newman-Keuls post-hoc analysis, ${ }^{*} \mathrm{p}<0.05, \mathrm{n}=6$ per treatment group. 
Citation: Reneer DV, Crowdus CA, Ghoshal S, Corkins J, Hisel RD, et al. (2014) Extent of Cerebrovascular Disruption Following Blast Exposure is Influenced by the Duration of the Positive Phase in Addition to Peak Overpressure. J Neurol Neurophysiol 5: 188. doi:10.4172/21559562.1000188

A.

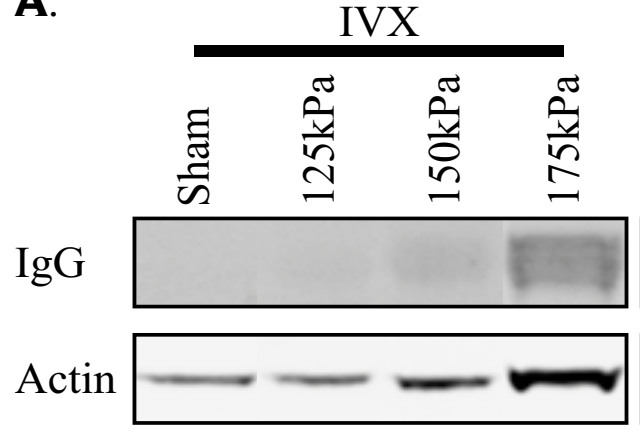

B

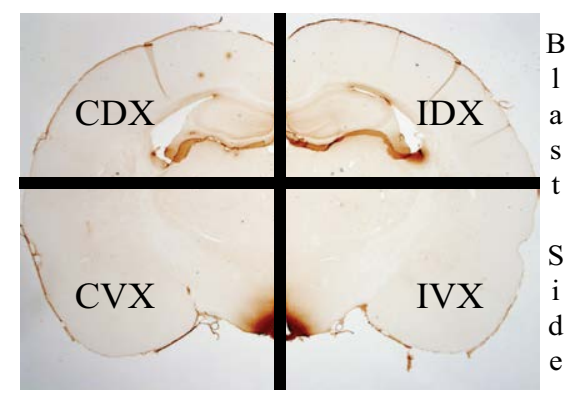

D

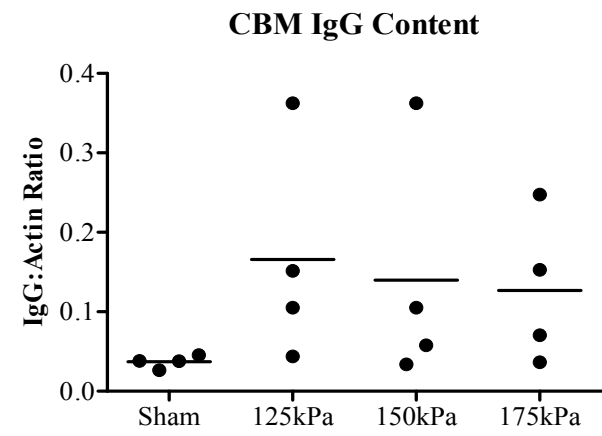

C

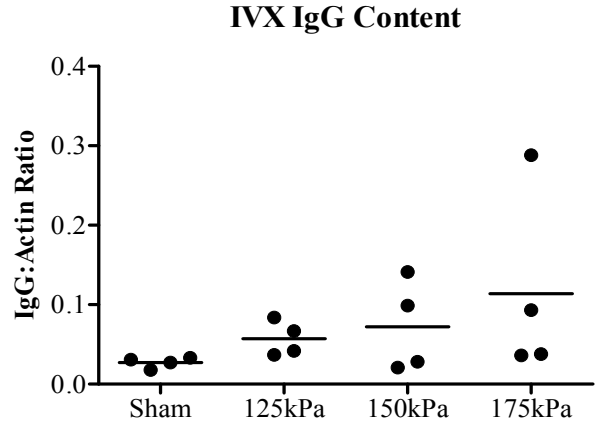

E

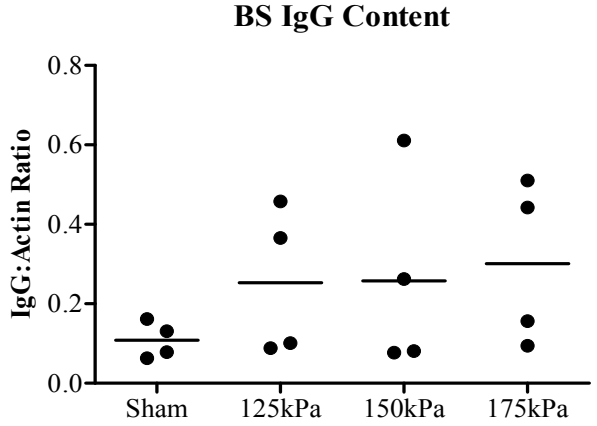

Figure 4: Blast overpressure dose-response following compressed-air driven blast exposure. (A) Representative bands from Western blots used to quantify lgG levels in the ipsilateral ventral cortex (IVX), cerebellum (CBM), and brain stem (BS). Samples were separated by electrophoresis in an order alternative to the one presented here to decrease inter-lane variability, however all blots shown here were imaged concurrently, and cropped to be repositioned as shown here. No other image modifications were made. (B) Diagram showing location of cuts made to separate cerebrum into ipsilateral ventral cortex (IVX), contralateral ventral cortex (CVX), ipsilateral dorsal cortex (IDX), and contralateral dorsal cortex (CDX). (C) Average IgG levels compared to actin levels in the IVX of animals exposed to increasing blast overpressure. (D) Average IgG levels compared to actin levels in the CBM of animals exposed to increasing blast overpressure. $€$. Average IgG levels compared to actin levels in the BS of animals exposed to increasing blast overpressure. One-way ANOVA, $n=4$ per treatment group.

\section{IgG extravasation as a function of pressure or impulse}

Comparison of IgG:Actin ratios as measured by Western blot analysis revealed a positive correlation between the total amount of IgG present in the IVX region and the amount of peak overpressure to which individual rats were exposed (Figure 5). Additionally, there appears to be a threshold effect at approximately $150 \mathrm{kPa}$ peak pressure. Animals exhibiting IgG:Actin ratios above 0.1 were mostly exposed to a peak overpressure of $150 \mathrm{kPa}$ or greater (Figure 5). There was no positive correlation between the impulse to which the animals were exposed and the IgG: Actin ratios these animals exhibited either when considered within an individual shockwave source (Figure 5B) or regardless of source (Figure 5D). No statistically significant relationship between peak overpressure or impulse exposure and IgG extravasation was found in the cerebellum or the brain stem (results not shown).

\section{Head rotation}

Head rotation due to blast was examined via high-speed (300 frames per second) video recording of head movement during blast exposure. Though there were significant differences between oxyhydrogen and compressed air in both the velocity of shockwaves and the amount of kinetic energy contained within the shockwaves [9], there was no significant difference in the total angle through which the head moved or in the average velocity of head movement between shockwave sources. 
A

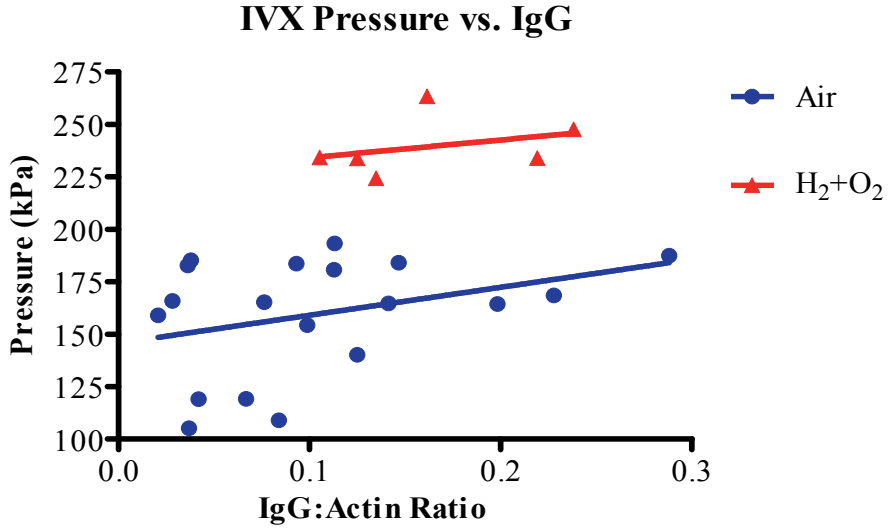

C

IVX Pressure vs. IgG

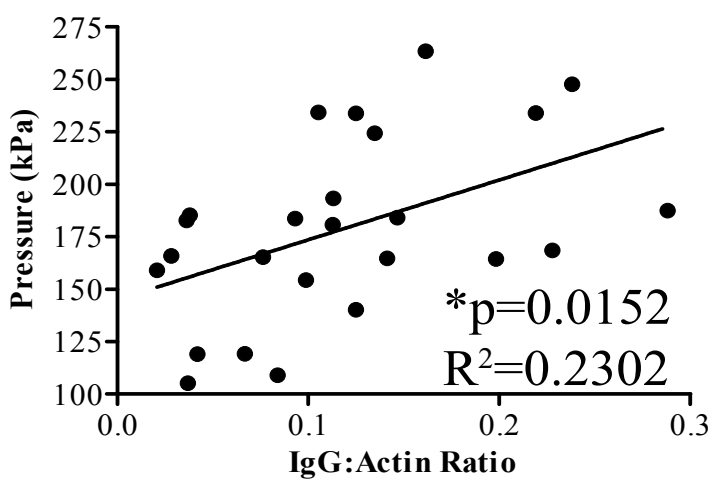

B

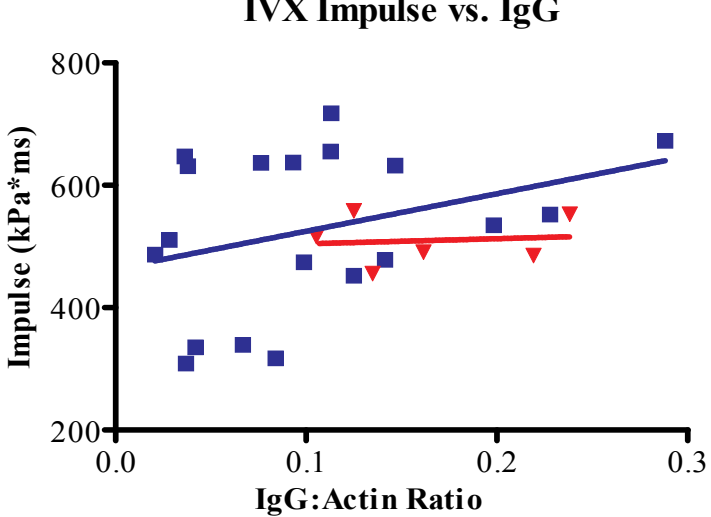

D

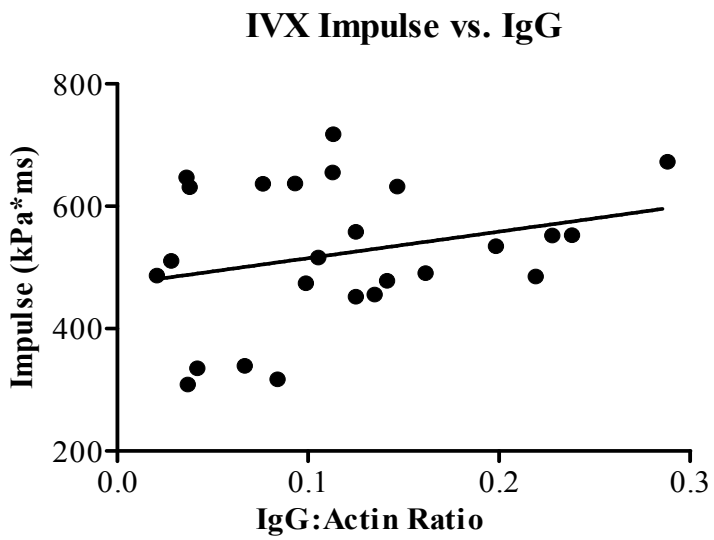

Figure 5: IVX IgG:Actin ratios as a function of peak overpressure and impulse exposure. (A) Peak blast overpressure exposure vs. IgG:Actin ratio separated by shockwave source. There appears to be a threshold effect whereby animals exhibiting lgG:Actin ratios above 0.1 were also mostly exposed to peak overpressures of $150 \mathrm{kPa}$ or greater. (B) Peak blast impulse exposure vs. IgG:Actin ratio separated by shockwave source. There was no positive effect of impulse on lgG extravasation for animals exposed to compressed air- or oxyhydrogen-driven shockwaves. (C) Peak blast overpressure exposure vs. IgG:Actin ratio. A positive correlation between the amount of IgG as detected by Western blot analysis and the peak overpressure to which the animals were exposed was evident. (D) Peak blast impulse exposure vs. IgG:Actin ratio. Though there was a general positive trend in the relationship between impulse and IgG extravasation, the relationship was not statistically significant. Pearson correlation, two-tailed. $n=6$ or 19 (A and B) or $n=25$ (C and D) per treatment group.

\section{Discussion}

Hematoma has been reported in both human case studies of blast injury [19-22] and animal models of bTBI [23]. Overt presence of hematoma precludes a diagnosis of mild TBI [24], however the cognitive squeal following blast exposure of magnitudes utilized in this study are more similar to a mild injury $[25,26]$. Mice exposed to blasts of similar peak overpressure exhibited mild deficits on spatial recognition tasks and rotarod measurements of motor coordination [25]. Furthermore, food consumption and exercise performance studies have suggested mild behavioral changes associated with memory, motor, and motivational activities [26]. Regardless of behavioral findings of other researchers using blasts of similar overpressure to those utilized in this study, the size of hematoma present in compressed air-driven shockwave exposed animals is substantial and, when accounting for size scale, would result in more significant behavioral deficits in humans with hematomas of similar size. Differences in shockwave physics between the sources used in this study could account for the differential hematoma profile. Given the dependency of the brain on cerebrovasculature, a disruption in the blood supply of the magnitude that would result from hematoma of this size would cause significant behavioral problems in individuals exposed to blast $[15,27,28]$.

Blood-brain barrier disruption due to compressed air-driven shockwave exposure was centered around the inferior cortex on the side of the brain that was first impacted by blast. This contrasts to previous studies where IgG was detected in the contralateral cortex and cerebellum following blast injury [17,29], or equally distributed in both hemispheres $[13,30]$. Some models of blast injury utilized rigid animal holding mechanisms that could have contributed to the brain injury through tertiary means $[17,29]$. In the present study, the placement of the animals in a plastic netting allowed some movement following blast exposure but prevented the head from 15 interacting with rigid surfaces [9]. This mechanism does allow for some head movement during blast exposure, resulting in the possibility that head rotation and acceleration/deceleration contributed to the observed pathology [15]. However, we did not observe differences in the extent or velocity of head movement with the two blast sources.

Though we were unable to generate a dose-response effect in terms of significantly higher amounts of IgG extravasation with increasing 
Citation: Reneer DV, Crowdus CA, Ghoshal S, Corkins J, Hisel RD, et al. (2014) Extent of Cerebrovascular Disruption Following Blast Exposure is Influenced by the Duration of the Positive Phase in Addition to Peak Overpressure. J Neurol Neurophysiol 5: 188. doi:10.4172/21559562.1000188

blast overpressure in the air-driven mode, IgG extravasation in the IVX was found to positively correlate with the peak pressure to which the animals were exposed. IgG extravasations, hematoma and petechial hemorrhages were more exaggerated in animals exposed to compressed air-driven shockwaves than in animals exposed to oxyhydrogen-driven shockwaves, even though the average peak overpressure of compressed air driven shockwaves was approximately $75 \mathrm{kPa}$ lower than that of oxyhydrogen-driven shockwaves. Taken together, these data suggest that both peak pressure and positive phase duration play a role in the cerebrovascular response to blast injury.

Previous studies in rats and larger animals have reported blood-brain barrier disruptions or vascular changes following blast overpressure $[13,17,29,30]$ or chemical explosive-induced brain injury $[31,32]$, however the cerebrovascular response to shockwaves from multiple sources with varying pressure-time signatures was not previously examined. Overpressure durations in previous bTBI studies vary from $0.3 \mathrm{~ms}$ to $52 \mathrm{~ms}[30,33,34]$, while overpressure durations in the present study varied from $3.2 \mathrm{~ms}$ to $5.3 \mathrm{~ms}$. When compared to the vast overpressure durations reported in previous bTBI studies, our data suggests a small increase in positive phase duration can have a substantial impact on the cerebrovascular response.

Certain environments, such as building or vehicle enclosures, common in battlefield situations could give rise to increased positive phase duration exposure and could result in exacerbated injury characteristics from a blast that may not have caused such injury in an open environment. The findings of this study suggest that positive phase duration should be considered when evaluating the extent and type of brain injuries sustained due to blast. Additionally, in addition to reducing the amount of blast pressure allowed through protective armor, engineers and designers of blast wave mitigation technologies may wish to consider strategies to shorten the positive phase duration of the shockwave or to attenuate the lengthening of the positive phase caused by reflection from solid surfaces.

\section{Acknowledgements}

This research was supported by NIH Grants F31NS074678 and P30NS051220, and by the Kentucky Spinal Cord and Head Injury Research Trust.

\section{References}

1. Lopez NE, Krzyzaniak MJ, Blow C, Putnam J, Ortiz-Pomales Y, et al. (2012) Ghrelin prevents disruption of the blood-brain barrier after traumatic brain injury. J Neurotrauma 29: 385-393.

2. Unterberg AW, Stover J, Kress B, Kiening KL (2004) Edema and brain trauma. Neuroscience 129: 1021-1029.

3. Schmidt RH, Grady MS (1993) Regional patterns of blood-brain barrier breakdown following central and lateral fluid percussion injury in rodents. J Neurotrauma 10: 415-430.

4. McIntosh TK, Vink R, Noble L, Yamakami I, Fernyak S, et al. (1989) Traumatic brain injury in the rat: characterization of a lateral fluid-percussion model. Neuroscience 28: 233-244.

5. Tanno H, Nockels RP, Pitts LH, Noble LJ (1992) Breakdown of the blood-brain barrier after fluid percussion brain injury in the rat: Part 2: Effect of hypoxia on permeability to plasma proteins. J Neurotrauma 9: 335-347.

6. Cherian L, Robertson CS, Contant CF Jr, Bryan RM Jr (1994) Lateral cortical impact injury in rats: cerebrovascular effects of varying depth of cortical deformation and impact velocity. J Neurotrauma 11: 573-585.

7. Moochhala SM, Md S, Lu J, Teng CH, Greengrass C (2004) Neuroprotective role of aminoguanidine in behavioral changes after blast injury. J Trauma 56: 393-403.

8. Kato K, Fujimura M, Nakagawa A, Saito A, Ohki T, et al. (2007) Pressuredependent effect of shock waves on rat brain: induction of neuronal apoptosis mediated by a caspase-dependent pathway. J Neurosurg 106: 667-676.
9. Reneer DV, Hisel RD, Hoffman JM, Kryscio RJ, Lusk BT, et al. (2011) A multimode shock tube for investigation of blast-induced traumatic brain injury. $J$ Neurotrauma 28: 95-104

10. Hyde DW (2004) CONWEP 2.1.0.8, Conventional Weapons Effects Program U.S.A.C.o. Engineers, Vicksburg MS (ed.).

11. Bass CR, Panzer MB, Rafaels KA, Wood G, Shridharani J, et al. (2012) Brain injuries from blast. Ann Biomed Eng 40: 185-202.

12. Bell MK (2008) Standardized model is needed to study the neurological effects of primary blast wave exposure. Mil Med 173: v-viii.

13. Skotak M, Wang F, Alai A, Holmberg A, Harris S, et al. (2013) Rat injury mode under controlled field-relevant primary blast conditions: acute response to a wide range of peak overpressures. J Neurotrauma 30: 1147-1160.

14. Sundaramurthy A, Alai A, Ganpule S, Holmberg A, Plougonven E, et al (2012) Blast-induced biomechanical loading of the rat: an experimental and anatomically accurate computational blast injury model. J Neurotrauma 29 . 2352-2364.

15. Goldstein LE, Fisher AM, Tagge CA, Zhang XL, Velisek L, et al. (2012) Chronic traumatic encephalopathy in blast-exposed military veterans and a blast neurotrauma mouse model. Sci Transl Med 4: 134ra60.

16. Garman RH, Jenkins LW, Switzer RC 3rd, Bauman RA, Tong LC, et al. (2011) Blast exposure in rats with body shielding is characterized primarily by diffuse axonal injury. J Neurotrauma 28: 947-959.

17. Readnower RD, Chavko M, Adeeb S, Conroy MD, Pauly JR, et al. (2010) Increase in blood-brain barrier permeability, oxidative stress, and activated microglia in a rat model of blast-induced traumatic brain injury. $J$ Neurosci Res 88: 3530-3539.

18. Long JB, Bentley TL, Wessner KA, Cerone C, Sweeney S, et al. (2009) Blast overpressure in rats: recreating a battlefield injury in the laboratory. Neurotrauma 26: 827-840.

19. Levi L, Borovich B, Guilburd JN, Grushkiewicz I, Lemberger A, et al. (1990) Wartime neurosurgical experience in Lebanon, 1982-85. I: Penetrating craniocerebral injuries. Isr J Med Sci 26: 548-554.

20. Murthy JM, Chopra JS, Gulati DR (1979) Subdural hematoma in an adult following a blast injury. Case report. J Neurosurg 50: 260-261.

21. Quintana DA, Parker JR, Jordan FB, Tuggle DW, Mantor PC, et al. (1997) The spectrum of pediatric injuries after a bomb blast. J Pediatr Surg 32: 307-310.

22. Scott BA, Fletcher JR, Pulliam MW, Harris RD (1986) The Beirut terrorist bombing. Neurosurgery 18: 107-110.

23. Kato K, Fujimura M, Nakagawa A, Saito A, Ohki T, et al. (2007) Pressuredependent effect of shock waves on rat brain: induction of neuronal apoptosis mediated by a caspase-dependent pathway. J Neurosurg 106: 667-676.

24. Krainin BM, Forsten RD, Kotwal RS, Lutz RH, Guskiewicz KM (2011) Mild traumatic brain injury literature review and proposed changes to classification. J Spec Oper Med 11: 38-47.

25. Koliatsos VE, Cernak I, Xu L, Song Y, Savonenko A, et al. (2011) A mouse model of blast injury to brain: initial pathological, neuropathological, and behavioral characterization. J Neuropathol Exp Neurol 70: 399-416.

26. Bauman RA, Elsayed N, Petras JM, Widholm J (1997) Exposure to subletha blast overpressure reduces the food intake and exercise performance of rats. Toxicology 121: 65-79.

27. Bogdanova Y, Verfaellie M (2012) Cognitive sequelae of blast-induced traumatic brain injury: recovery and rehabilitation. Neuropsychol Rev 22: 4-20.

28. Luethcke CA, Bryan CJ, Morrow CE, Isler WC (2011) Comparison of concussive symptoms, cognitive performance, and psychological symptoms between acute blast-versus nonblastinduced mild traumatic brain injury. J Int Neuropsychol Soc 17: 36-45.

29. Kochanek PM, et al. (2009) Blast-induced traumatic brain injury and polytrauma-a critical problem begging for new insight and new therapies. J Neurotrauma.

30. Yeoh S, Bell ED, Monson KL (2013) Distribution of blood-brain barrier disruption in primary blast injury. Ann Biomed Eng 41: 2206-2214.

31. Bauman RA, Ling G, Tong L, Januszkiewicz A, Agoston D, et al. (2009) An introductory characterization of a combat-casualty-care relevant swine mode of closed head injury resulting from exposure to explosive blast. J Neurotrauma 26: 841-860. 
Citation: Reneer DV, Crowdus CA, Ghoshal S, Corkins J, Hisel RD, et al. (2014) Extent of Cerebrovascular Disruption Following Blast Exposure is Influenced by the Duration of the Positive Phase in Addition to Peak Overpressure. J Neurol Neurophysiol 5: 188. doi:10.4172/21559562.1000188

Page 8 of 8

32. Cheng J, Gu J, Ma Y, Yang T, Kuang Y, et al. (2010) Development of a rat model for studying blast-induced traumatic brain injury. J Neurol Sci 294: 23-28.

33. Cernak I, Wang Z, Jiang J, Bian X, Savic J (2001) Ultrastructural and functional characteristics of blast injury-induced neurotrauma. J Trauma 50: 695-706.
34. Cernak I, Wang Z, Jiang J, Bian X, Savic J (2001) Cognitive deficits following blast injury-induced neurotrauma: possible involvement of nitric oxide. Brain Inj 15: 593-612.

This article was originally published in a special issue, Traumatic Brain Injury: Diagnosis \& Treatment handled by Editor(s). Dr. Douglas Mckay, University of Miami, USA 